\title{
ESPIRITUALIDADE E SAÚDE DO IDOSO EM TEMPOS DE PANDEMIA: REFLEXÃO NA TEORIA DE JEAN WATSON
}

Tânia Maria de Oliva Menezes' ORCID: 0000-0001-5819-0570

Luciana Mitsue Sakano Niwa" ORCID: 0000-0002-9342-7454

Larissa Sapucaia F. Esteves ${ }^{\text {III }}$ ORCID: 0000-0003-3489-2599

Daniela Garcia Damaceno'v ORCID: 0000-0001-8656-009X

Márcia de Assunção Ferreirav ORCID: 0000-0002-6991-7066

Raul Fernando Guerrero Castañedavi ORCID: 0000-0003-3996-5208

'Universidade Federal da Bahia. Salvador, Bahia, Brasil.

"Universidade de São Paulo. São Paulo, São Paulo, Brasil.

"'Universidade do Oeste Paulista. Presidente Prudente, São Paulo, Brasil.

"Universidade de São Paulo. São Paulo, São Paulo, Brasil.

vniversidade Federal do Rio de Janeiro. Rio de Janeiro, Rio de Janeiro, Brasil.

"Universidad de Guanajuato. Celaya, Guanajuato, México.

Autor Correspondente: Tânia Maria de Oliva Menezes E-mail: tomenezes50@gmail.com

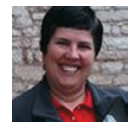

Como citar:

Menezes TMO, Niwa LMS, Esteves LSF, Damaceno DG, Ferreira MA, Guerrero-Castañeda RF. Espiritualidade e Saúde do Idoso em Tempos de Pandemia: Reflexão na Teoria de Jean Watson. In: Santana RF (Org.). Enfermagem gerontológica no cuidado do idoso em tempos da COVID 19. Brasilia, DF: Editora ABen; 2021. 171 p. (Serie Enfermagem e Pandemias, 5). https://doi.org/10.51234/aben.21.e05.c14

\section{INTRODUÇÃO}

Vivemos em tempos difíceis desde o primeiro caso da COVID-19, doença causada pelo novo coronavírus (Sars-Cov-2), notificado em Wuhan, China, em dezembro de 2019. Em poucos meses, a nova doença se espalhou por todo o mundo chegando ao Brasil em 26 de fevereiro de 2020.

A Organização Mundial da Saúde (OMS, 2020) declarou em 11 de março de 2020 pandemia de COVID-19, pela rápida disseminação geográfica e pelo nível alarmante de contaminação. Na primeira quinzena de junho de 2020 foram 7.410.510 casos confirmados e 418.294 mortes pela doença, no mundo (1). O Brasil lidera o segundo lugar no ranking mundial, com 850.514 casos confirmados e 42.720 mortes por COVID-19(2).

No Brasil, 69\% das mortes por COVID-19 são de pessoas idosas ${ }^{(3)}$. A diminuição da eficácia do sistema imune provocada pela fisiologia do envelhecimento e doenças crônicas potencialmente incapacitantes tornam as pessoas idosas as principais vítimas e grupo de risco da doença.

Medidas sanitárias e de distanciamento social têm sido eficazes para o controle dos casos, evitando falência dos equipamentos de saúde. Apesar do esforço científico, ainda não há cura e vacina para esta doença. A mudança no estilo de vida imposta pela pandemia da COVID-19 nos traz desafios com a saúde em sua totalidade.

O ser humano possui dimensões que se integram, formando um todo biopsicosocioespiritual, imerso em uma dada cultura ${ }^{(4,5)}$. Nesse ínterim, cuidar da saúde humana nos desafia a atender a pessoa de forma holística, nestas dimensões. Nesse sentido, destaca-se que a enfermagem orienta os cuidados para a pessoa na intenção de manter e promover a saúde, concebendo-a de forma holística; logo, os cuidados devem atender às diferentes dimensões humanas e, com isso, transcender a materialidade biológica do ser, às demandas físicas, e requerer atenção às outras dimensões. 
O ser humano expressa a cultura em que está imerso, e por isso, a dimensão sociocultural toma vulto nas questões de saúde. Não menos importante tem-se a dimensão psíquica e a espiritualidade, que também são importantes, quando se consideram as questões do cuidado à saúde humana. Neste contexto, a dimensão espiritual se configura em uma área promissora de pesquisa para profissionais de saúde, em especial na enfermagem gerontológica, e se reveste de crucial importância quando se relevam os cuidados de enfermagem à pessoa idosa.

A exploração do tema no campo acadêmico e científico é crucial para que se estabeleçam as relações entre a espiritualidade e a saúde, por isso são necessárias reflexões e pesquisas sobre o tema. Refletir sobre o papel da espiritualidade no conforto, bem-estar e na felicidade das pessoas contribui para a oferta de cuidados mais aplicados às necessidades dos usuários dos serviços de enfermagem.

Tendo em vista a dimensão espiritual na constituição do ser e o papel que a sua expressão - a espiritualidade - ocupa na vida/existência humana, evidenciada em estudos e pesquisas, se ressalta que na situação atual em que o mundo vivencia uma grave crise sanitária com a pandemia da COVID-19, causada por um vírus com alto poder de transmissibilidade, não se deve negligenciar a espiritualidade no cuidado às pessoas, em especial aos idosos. Isto porque esta faixa etária figura entre o grupo suscetível à infecção e a desenvolverem complicações, com alto índice de morte ${ }^{(6)}$.

Esta situação de vulnerabilidade fragiliza ainda mais os idosos e requer da enfermagem prontidão para cuidar e aliviar seus sofrimentos, instilando-Ihes a esperança e a fé, com estratégias de cuidado que possam auxiliá-los no enfrentamento desta adversidade. E nesse propósito, dar atenção à espiritualidade e às suas expressões no atendimento das demandas dos idosos é condição para cuidá-lo em uma perspectiva holística, integradora, aliando o afeto, que se configura em um diferencial na qualidade do cuidado de enfermagem.

Em tempos de pandemia, a espiritualidade tem uma força curativa que potencializa ações como amor a vida, perdão, fraternidade, solidariedade, misericórdia e justiça. Ao mesmo tempo, permite a convicção de que a realidade captada é maior do que as análises nos dizem. Ela permite crer que o invisível e o impossível são partes do visível e do previsível.

Considerando que a pandemia pela COVID-19 mudou o estilo de vida, especialmente das pessoas idosas; o aumento dos casos e a falta de estratégias de controle no Brasil, é necessário cuidar do indivíduo em sua totalidade, e para tal, se faz imprescindível refletir a respeito da espiritualidade, sobre como os idosos têm buscado e vivido a espiritualidade e como os enfermeiros têm encorajado tais ações.

\section{OBJETIVO}

Refletir sobre espiritualidade e saúde do idoso em tempos de pandemia.

\section{MÉTODO}

Ensaio teórico de natureza reflexiva sobre espiritualidade e cuidados à saúde da pessoa idosa, baseado em conceitos e em uma teoria de enfermagem, publicados em artigos científicos nacionais e internacionais, a partir de dois eixos temáticos: 1. Religiosidade e espiritualidade, conceitos e relações com a saúde e práticas de cuidado; e o cuidado espiritual à pessoa idosa fundamentado na Teoria de Jean Watson.

\section{RESULTADOS}

\section{Religiosidade e espiritualidade: conceitos e relações com a saúde e práticas de cuidado}

Definições e conceitos de espiritualidade vêm sofrendo transformações e se afastando dos conceitos de religiosidade, expandindo-se para abarcar o bem-estar, a felicidade, os propósitos de vida ${ }^{(7)}$. Nesta direção, tem-se os estudos de Viktor Frankl, que destaca a capacidade do ser humano de se autotranscender, fazer os enfrentamentos necessários à vida e seguir na busca de significados e sentidos para a sua existência ${ }^{(8)}$. 
Apesar de serem, algumas vezes, tratadas como sinônimos, a religiosidade e a espiritualidade apresentam diferenças essenciais em seu significado real e a sua influência para o cuidado em saúde. De uma forma geral, a espiritualidade é a relação e afinidade estabelecida entre um sujeito e um ser superior; já a religiosidade é reconhecida como a adesão a práticas e crenças de uma instituição religiosa ${ }^{(9)}$.

A espiritualidade seria um fator interno que influencia na percepção de sentido na vida e estudos evidenciam esta vertente, a da espiritualidade relacionada a propósitos, a sentidos e significados atribuídos à vida e à existência, que é importante na fase da velhice, uma vez que encontrar um sentido na vida auxilia no enfrentamento das perdas comuns no processo de envelhecimento ${ }^{(10)}$.

A espiritualidade pode ser entendida, então, como uma busca subjetiva e individual da compreensão do sentido da vida, sua terminalidade e relação com o sagrado/superior. A partir dessa compreensão, o indivíduo vivencia os diferentes fenômenos transcendendo sua existência física, podendo ou não assumir práticas religiosas formais e formação de comunidade ${ }^{(11)}$. Assim, ela acontece independentemente de religião e crenças, assumindo um sentido amplo de (res)significação dos sentimentos, experiências e enfrentamento de situações críticas ${ }^{(9)}$.

Por outro lado, a religiosidade é reconhecida como uma expressão da espiritualidade de pessoas que desenvolvem atividades em relação às suas crenças ${ }^{(12)}$. Expressa comportamentos, valores, rituais, doutrinas e características sociais comuns que representam uma dimensão social e cultural, podendo ser intrínseca (vivência pessoal) ou extrínseca (vivência social) ${ }^{(9,11)}$.

Na prática assistencial, é importante que os profissionais de enfermagem estejam atentos às expressões das pessoas confiadas aos seus cuidados, em particular às suas culturas. Compreender a cultura dos grupos humanos é crucial para que se tenha bons resultados no atendimento ${ }^{(13)}$. Tendo em vista as relações possíveis entre as culturas, as religiões, as práticas e expressões de religiosidade e espiritualidade, considerar a diversidade humana é relevante nas discussões sobre promoção da saúde e práticas de cuidado(14).

Para garantir um cuidado centrado nas diferenças e na diversidade, o enfermeiro precisa transcender as suas próprias crenças, valores e culturas, atender as demandas de ordem biológicas, sociais, comportamentais e espirituais das pessoas a quem cuida, em especial as pessoas idosas, quando se considera as particularidades de cenários de inseguranças postas por situações críticas, como no caso de uma pandemia.

Quando se alia o envelhecimento à espiritualidade, observa-se relação entre a dimensão espiritual e a qualidade de vida de pessoas idosas, ajudando-as a suportar as limitações que o envelhecimento traz no enfrentamento das adversidades ${ }^{(15)}$.

Com relação aos idosos longevos, com 80 anos ou mais, pesquisas têm mostrado que a espiritualidade e a religiosidade têm papel fundamental na percepção do envelhecimento como privilégio e dádiva divina, cuja fé em Deus repousa o significado à vida ${ }^{(16)}$, e também na expressão da resiliência, que contribui para que enfrentem situações que lhes prejudicam o bem-estar ${ }^{(17)}$.

Em razão disso, articular uma teoria de enfermagem na proposição de cuidados espirituais atende ao movimento acadêmico e científico, que tem se mostrado promissor no campo da saúde, em particular no contexto atual da pandemia de Covid-19, que tanto tem preocupado e ameaçado a saúde e a qualidade de vida das pessoas em geral, e dos idosos, em especial.

\section{Cuidado espiritual à pessoa idosa fundamentado na Teoria de Jean Watson}

Jean Watson em sua teoria do Cuidado Humano reúne elementos fundamentais para integrar o cuidado espiritual. Em seus conceitos, ela aponta que o cuidado é um fenômeno que vai além da hospitalização e cura, pois é uma interação entre dois seres humanos, a enfermeira e a pessoa cuidada. Sua teoria tem bases fenomenológicas e existencialistas ${ }^{(18)}$, o que permite a integração das artes e humanidades na consciência estética da enfermeira.

Por esta razão, o processo de cuidar vai muito além de um momento clínico, não se reduz às condições da terapia médica, mas contempla o ser humano em um campo transpessoal. Para o enfermeiro, o ideal 
no cuidado é conhecer o outro, criar um campo fenomenológico de cuidado, um campo de interação para conhecê-lo. O cuidado espiritual é uma extensão dos cuidados de enfermagem ${ }^{(19)}$, podendo ser promovido e capacitado para a residência do idoso, pois é o enfermeiro que motiva a pessoa a ser cuidada (o idoso) no cuidado, ajudando-o a descobrir seu potencial e seu próprio ser.

Especial ênfase é dada à compreensão do outro, à empatia, às relações inter e transpessoais, que permitem estabelecer a comunicação, envolver o outro no momento do cuidado e ajudá-los a descobrir formas de interagir consigo mesmos, com o mundo e com sua divindade.

No Processo Clinical Caritas, Watson aborda a valorização do ser humano além da dimensão física ${ }^{(19)}$, por isso, pode-se dizer que é um cuidado espiritual, pois atende a razões estéticas e metafísicas, que muitas vezes dependem das crenças das pessoas cuidadas e são elas que dão sentido à sua vida, a enfermeira procura respeitar essas crenças e suas formas mais complexas entrando no mundo da pessoa.

Baseada nos conceitos de espiritualidade revistos, esta teoria aborda que o cuidado é precisamente um ato sagrado, pois o ser humano é parte do universo e, portanto, um ser divino, por isso a enfermeira pode ir além do sentido profissional e estabelecer uma relação que permita com sensibilidade, empatia e amor cuidar do outro, dentro destes valores humanos é que o cuidado é um cuidado espiritual.

Nos 10 processos clínicos, propostos por Watson, dois relacionados à espiritualidade se destacam: o Processo 3, que menciona que a enfermeira promove o cultivo da sua própria prática espiritual e a do outro na relação transpessoal, e o Processo 10, ao enfatizar que a enfermeira deve se abrir aos mistérios e dimensões espirituais da vida e da morte, para cuidar de sua alma ou espírito e da alma da pessoa que está sendo cuidada ${ }^{(5,19)}$.

Neste sentido, o cuidado espiritual é muito mais profundo do que apenas dizer que o cuidado da enfermeira é físico, emocional/mental, social e espiritual. Ao atender a todas as dimensões do idoso que está sendo cuidado, o cuidado espiritual permite que a outra pessoa seja envolvida em ajudá-la a se conhecer, a encontrar em suas crenças ou divindade a força para enfrentar momentos difíceis, como o que ela está vivenciando atualmente. E quanto ao isolamento espiritual, este seria uma oportunidade de se encontrar e redescobrir o sentido vital da existência com a sabedoria dos anos vividos.

É importante lembrar que o idoso pode estar em casa, em uma Instituição de Longa Permanência para Idosos ou hospitalizado, portanto, o cuidado se estende nestes cenários e qualquer que seja a situação de convivência, o cuidado espiritual ajuda no processo de sentir-se acompanhado, exaltando e conectando-se com o que o idoso considera divino e que dá sentido ao seu momento de vida.

Dentro de algumas estratégias, as crenças e valores do idoso, suas expectativas, suas conexões mais profundas com a vida devem primeiro ser identificadas. Esse primeiro reconhecimento é importante para encontrar o caminho do acompanhamento. A enfermeira compreende que nem todos os idosos têm a mesma crença que ela. Nesse sentido, o cuidado espiritual recomenda o respeito às crenças, com uma atitude de empatia para com o idoso, não procura impor, ou mesmo, praticar a mesma crença que o idoso, procura compreendê-lo e apoiá-lo em sua prática.

Os cenários podem favorecer ou limitar a prática espiritual, se ela for concebida a partir das práticas religiosas, porém, deve-se buscar nos cuidados um ambiente que favoreça essa prática em termos de tempo e espaço. Quando acompanhada por outros idosos, deve-se contemplar a variedade de crenças que possam existir.

O cuidado espiritual se apresenta através da oração, música, leitura de textos relacionados com as crenças, meditação. Esta última, às vezes leva a visão de ser praticada apenas pelas religiões orientais, porém, é um momento de quietude e harmonia de estar no encontro com o universo, é uma forma de ouvir o que há no vazio da vida. Também o cuidado espiritual da enfermeira a pessoa idosa se dá através do estímulo a fé, a conexão com um ser superior e ao exercício da religiosidade intrínseca.

No contexto situacional da pandemia da COVID-19, em que o distanciamento social é uma medida protetiva, se a pessoa tiver acesso à tecnologia, pode fazer as videochamadas com sua família, amigos ou pessoas que 
compartilham sua fé. Às vezes, a ligação pelo telefone convencional dá aquele sentimento de companhia, ao ouvir a voz daqueles que se amam e são significativos em sua vida.

É importante incentivar a busca da espiritualidade, e se for relacionado com a religião do idoso, perguntar se o apoio é apropriado e em que medida esse apoio pode ser dado. Os idosos valorizarão se o acompanhamento da enfermeira é necessário, ou se a enfermeira só fornece os meios apropriados para que os idosos possam encontrar seu próprio tempo e lugar.

Afeto e empatia são as bases para favorecer o cuidado espiritual. Os idosos devem entender que não é necessário a enfermeira ser mística para prestar esse cuidado, mas, devem estar conscientes de sua própria espiritualidade e de seu senso de vida pessoal e profissional. Quando a pessoa idosa consegue isso em seu dia a dia, ela desenvolve a consciência caritas, que lhe fornece ferramentas para promover o cuidado espiritual.

\section{Limitações}

Como limitação indica-se a circunscrição da reflexão em apenas uma teoria de enfermagem, devendo-se ser ampliada para outras teóricas, o que trará mais possibilidades para se pensar e propor cuidados que atendam à dimensão espiritual da pessoa idosa, à luz de outros modelos de cuidar.

\section{Contribuições para Enfermagem}

Ao se refletir sobre a espiritualidade e seu lugar na atenção aos idosos, em especial no contexto da pandemia da COVID-19, à luz da Teoria de Jean Watson, contribui-se para a prática do cuidado cientificamente fundamentado, como também, para o atendimento a uma necessidade humana peculiar que, se não for relevada, resulta em uma assistência fragmentada, comprometendo a qualidade do cuidado de enfermagem.

\section{CONSIDERAÇÕES FINAIS}

Tempos de pandemia são marcados pela separação física imposta pelo distanciamento social e por tantas mortes sem despedidas. Nesse sentido, à luz dos conceitos e teoria discutidos, orar e interceder uns pelos outros, promovendo encontros de fé por meio de orações, preces, rezas se configura em estratégias de cuidado que aproximam as pessoas, respeitadas suas crenças, culturas e filosofias de vida.

Cuidar de pessoas tratando de suas dores, segurando sua mão, ouvindo suas queixas, anseios, transmitindo segurança, conforto, alívio e ser o elo entre o paciente, família e equipe interdisciplinar são exemplos de atuação espiritual que podem nos passar despercebidos em razão das muitas atividades cotidianas. No entanto, o fazer diário pode ser facilitado/viabilizado quando o enfermeiro atua guiado por conceitos e teorias.

A enfermagem intercede pelas pessoas que estão sob seus cuidados quando atua em prol da beneficência e não maleficência, da ótima recuperação, quando transmite com sua chegada o conforto, instila fé, confiança e a segurança de que tudo o que é possível fazer estará sendo feito para que a pessoa cuidada fique bem, apesar de todas as adversidades.

\section{AGRADECIMENTO}

Associação Brasileira de Enfermagem e Departamento Científico de Enfermagem Gerontológica Nacional.

\section{REFERENCIAS}

1. Organização Pan-americana da Saúde (OPAS). Folha informativa-COVID-19. [Internet]. 2020. [cited 2020 Jun 12 ]. Available from: https://www.paho.org/bra/index.php?option=com_content\&view=article\&id=6101:covid19\&ltemid=875 
2. Organização mundial da Saúde (OMS). Pandemia da doença de coronavírus (COVID-19). [Internet]. 2020 [cited 2020 Jun 12]. Available from: https://www.who.int/emergencies/diseases/novel-coronavirus-2019

3. Ministério da Saúde. Boletim 15. Boletim Epidemiológico Especial(COE-COVID-19). [Internet]. 2020. [cited 2020 Jun 10]. Available from: https://portalarquivos.saude.gov.br/images/pdf/2020/May/09/2020-05-06-BEE15-Boletim-do-COE.pdf

4. Leininger M. Culture Care Theory: a major contribution to Advance Transcultural Nursing Knowledge and Practices. J Transcult Nurs. 2002;13(3):189-92. https://doi.org/10.1177/10459602013003005

5. Watson J. Human Caring Science: a theory of nursing. Boston: Jones and Bartlett; 2011.

6. Lima CFM, Ferreira MA. Práticas integrativas e complementares de saúde no enfrentamento da pandemia COVID-19. In: Enfermagem gerontológica no cuidado do idoso em tempo da COVID 19 / Associação Brasileira de Enfermagem. Brasília, DF: ABen/DCEG, 2020. p. 74-79. (Serie enfermagem e pandemias).

7. Koenig HG. Concerns About Measuring "Spirituality" in Research. J Nerv Ment Dis. 2008;196(5):349-355. https://doi. org/10.1097/NMD.0b013e31816ff796

8. Frankl V. Em busca de sentido: um psicólogo no campo de concentração. Petrópolis: Vozes, 2009.

9. Thiengo PCS, Gomes AMT, Mercês MC, Couto PLS, França LCM, Silva AN. Spirituality and religiosity in health care: An integrative review. Cogitare Enferm. 2019;24:e58692. https://doi.org/10.5380/ce.v24i0.58692.

10. Sommerhalder C. Sentido de vida na fase adulta e velhice. Psicol. Reflex. Crit. 2010; 23(2):270-277. https://doi.org/10.1590/ S0102-79722010000200009

11. Koenig HG, Mccullough M, Larson DB, editors. Handbook of religion and health: a century of research reviewed. New York: Oxford University Press; 2001

12. Valente TCO, Quelho CT, Cavalcanti APR, Carmo HO. Espiritualidade, religiosidade e saúde: velhos debates, novas perspectivas. Interações. 2016:11(20):85-87. https://doi.org/10.5752/P.1983-2478.2016v11n20p85

13. Gaspar AMFCC, Branco CB, Pedro CFS, Nunes DF, Alves NSA, Reis A. As estratégias de enfermagem adotadas para ultrapassar as barreiras culturais e linguísticas com pessoas culturalmente diversas: uma scoping review. Rev UIIPS. 2020;8(1):215-222. https://doi.org/10.25746/ruiips.v8.i1.19893

14. Timm V. Religious culture and health promotion: care, practice, object. Rev Bras Promoç Saúde. 2015;28(2):151-152. https:// doi.org/10.5020/18061230.2015.p149

15. Dias EN, Pais-Ribeiro JL. Espiritualidade e qualidade de vida de pessoas idosas: um estudo relacional. Psicol Saúde Doenças. 2018;19(3):591-604. https://doi.org/10.15309/18psd190310

16. Marinho M, Chaves R, Gomes J, Reis L. Longevidade e espiritualidade: o envelhecer como uma dádiva de Deus. RBCEH. 2018;14(2):159-168. https://doi.org/10.5335/rbceh.v14i2.6702

17. Reis LA, Menezes TMO. Religiosity and spirituality as resilience strategies among long-living older adults in their daily lives. Rev Bras Enferm. 2017;70(4):761-6. [Thematic Edition "Good Practices: Fundamentals of care in Gerontological Nursing"]. https://doi.org/10.1590/0034-7167-2016-0630

18. Watson J. Nursing: the philosophy and science of caring. Boulder: University Press of Colorado; 2008.

19. Costa JR, Arruda GO, Barreto MS, Serafim D, Sales CA, Marcon SS. Nursing professionals' day-to-day and Jean Watson's Clinical Caritas Process: a relationship. Rev Enferm UERJ. 2019;27:e37744. https://doi.org/10.12957/reuerj.2019.37744 